\title{
A NOTE ON FINITE DIMENSIONAL SUBRINGS OF POLYNOMIAL RINGS
}

\author{
PAUL EAKIN
}

\begin{abstract}
Let $k$ be a field and $\left\{X_{\alpha}\right\}_{\alpha \in \Delta}$ a family of indeterminates over $k$. We show that if $A$ is a ring of Krull dimension $d$ such that $k \subseteq A \subseteq k\left[\left\{A_{\alpha}\right\}_{\alpha \in \Delta}\right]$ then there are elements $Y_{1}, \cdots, Y_{d}$ which are algebraically independent over $k$ and a $k$-isomorphism $\phi$ such that $k \subseteq \phi(A) \subseteq k\left[Y_{1}, \cdots, Y_{d}\right]$. This is used to show that a onedimensional ring $A$ which satisfies the above conditions is necessarily an affine ring over $k$ and is necessarily a polynomial ring if it is normal. In addition we show that such a ring $A$ is a normal affine ring of transcendence degree two over $k$ if and only if it is a two-dimensional Krull ring such that each essential valuation of $A$ has residue field transcendental over $k$.
\end{abstract}

Introduction. In [EZ] Evyatar and Zaks ask whether a Dedekind domain which is caught between a field $k$ and the polynomials in a finite number of variables over $k$ is necessarily a polynomial ring. Here we show that the answer is affirmative with no restriction on the number of variables and without assuming the ring satisfies all three of the Noether axioms on a Dedekind domain (it need only be one dimensional and integrally closed). In addition we give some general results which relate subrings of the polynomials over a field to noetherian rings.

The author is indebted to W. J. Heinzer for the benefit of many stimulating conversations on this subject and to Kenneth Kubota for a suggestion which simplified the proof of Lemma B.

1. In all that follows, a ring will always be assumed to be commutative and to possess a multiplicative identity $1 \neq 0$. By dimension $A$ we always refer to the Krull dimension of the ring $A$.

Lemma A (Reduction to the case of a finite number of variables). Let $D$ be an integral domain and $\left\{X_{\alpha}\right\}_{\alpha \in \Delta}$ a family of indeterminates over $D$. Let $A$ be a ring such that

(1) $D \leqq A \leqq D\left[\left\{X_{\alpha}\right\}_{\alpha \in \Delta}\right]$.

(2) A satisfies the d.c.c. on prime ideals.

Received by the editors March 3, 1971 .

AMS 1969 subject classifications. Primary 1635, 1230, 1615.

Key words and phrases. Dedekind domain, Krull ring, polynomial ring, affine ring, noetherian ring, Krull dimension. 
Then there exist a finite number of indeterminates $\left\{Y_{i}\right\}_{i=1}^{k}$ and a D-homomorphism $\phi$ such that $D\left[\left\{X_{\alpha}\right\}_{\alpha \in \Delta}\right] \stackrel{\phi}{\rightarrow} D\left[Y_{1}, \cdots, Y_{k}\right]$ and such that $\phi$ restricted to $A$ is an isomorphism.

Proof. Let $M_{0}$ denote the prime ideal of $R=D\left[\left\{X_{\alpha}\right\}_{\alpha \in \Delta}\right]$ generated by the collection $\left\{X_{\alpha}\right\}_{\alpha \in \Delta}$. Now let $P_{0}=M_{0} \cap A$ and, if possible, choose $f_{0} \in P_{0}$ such that $f_{0} \neq 0$. Now $f_{0}$ involves only a finite number of the variables say $X_{1}, \cdots, X_{t_{0}}$. Hence if $M_{1}$ is the prime of $R$ generated by all of the $X_{\alpha}$ except $\left\{X_{i}\right\}_{i=1}^{t_{0}}$ then $f_{0} \notin P_{1}=M_{1} \cap A$. Now if $P_{1} \neq 0$ choose a nonzero $f_{1} \in P_{2}$. As before, choose $f_{1} \in P_{2}$ and as $f_{1}$ involves only finitely many variables we extend our finite collection $\left\{X_{i}\right\}_{i=1}^{t_{0}}$ to the finite collection $\left\{X_{i}\right\}_{i=1}^{t_{1}}$ which includes those variables. We can proceed inductively and produce a chain of prime ideals $A>P_{0}>P_{1}>\cdots>P_{k}$ which must be finite by assumption. Thus $P_{k}=0$ for some $k$ and it follows that there is a finite collection of indeterminates $\left\{X_{1}, \cdots, X_{k}\right\}$ such that if $M_{k}$ is the prime ideal of $R$ generated by $\left\{X_{\alpha}\right\}_{\alpha \in \Delta} \backslash\left\{X_{1}, \cdots, X_{k}\right\}$ then $M_{k} \cap A=0$. Thus if we consider the natural $D$-homomorphism $A / M_{k} \cap A \stackrel{\phi}{\rightarrow} R / M_{k}$, within isomorphism we have

$$
D \leqq A \leqq D\left[\bar{X}_{1}, \cdots, \bar{X}_{k}\right] \text {. }
$$

Thus the $\bar{X}_{i}$ and $\phi$ satisfy the conclusion of the theorem.

LemMA B. Suppose $k$ is a field and $X_{1}, \cdots, X_{n}$ are indeterminates over $k$. Let $A$ be a ring such that $k \leqq A \leqq k\left[X_{1}, \cdots, X_{n}\right]$. Then $A$ is of dimension $d$ for some $d \leqq n$ and there exist $d$ indeterminates $Y_{1}, \cdots, Y_{d}$ and $a k$ homomorphism

$$
\phi: k\left[X_{1}, \cdots, X_{n}\right] \rightarrow k\left[Y_{1}, \cdots, Y_{d}\right]
$$

which is an isomorphism on $A$. If $k$ is an infinite field then by a linear change of variables one can find a choice of $X_{1}, \cdots, X_{n}$ such that the kernel of $\phi$ is the ideal $\left(X_{d+1}, \cdots, X_{n}\right)$.

Proof. That dimension $A \leqq n$ is well known: It is simply a consequence of the fact that every valuation overring of $A$ must extend to a $k$-valuation of $k\left(X_{1}, \cdots, X_{n}\right)$, and must consequently be of rank $\leqq n$ [ZS, p. 10, Corollary 1]. The assertion of the lemma will follow when we prove the following:

If $k \leqq A \leqq k\left[X_{1}, \cdots, X_{n}\right]$ and $A$ is of dimension $d<n$, then there exists an element $f \in k\left[X_{1}, \cdots, X_{n}\right]$ such that:

(a) for some $i, f=X_{i}-h\left(X_{1}, \cdots, X_{n}\right)$ and $h$ is independent of $X_{i}$,

(b) $(f) k\left[X_{1}, \cdots, X_{n}\right] \cap A=0$, and

(c) if $k$ is infinite then $f$ can be taken to be a linear combination of the $X_{i}$.

The proof is by induction on $n$. The case $n=1$ is obvious. Let $P$ be a prime ideal of $A$ which is minimal with respect to the following property: 
$P$ has the form $(f) \cap A$ for some $f \in k\left[X_{1}, \cdots, X_{n}\right]$ such that $f$ has form $X_{i}-h\left(X_{1}, \cdots, X_{n}\right)$ where $h$ is independent of $X_{i}$ (in case $k$ is infinite we ask that $h$ be linear). Since $A$ is finite dimensional, such a $P$ certainly exists and there is no loss in assuming $X_{i}=X_{n}$. Now for such $f$ we have that $k\left[X_{1}, \cdots, X_{n}\right] /(f)=k\left[\bar{X}_{1}, \cdots, \bar{X}_{n-1}\right]$ and by dimension theory the $X_{i}$ are algebraically independent over $k$ [ZS, Theorem 21, p. 195 and Theorem 25, p. 200]. We have $k \leqq A / p \leqq k\left[X_{1}, \cdots, X_{n-1}\right]$. Now if $P=0$ we are through. If $P \neq 0$ then it must be true that dimension $A / p<$ $n-1$ for dimension $A / p=n-1$ would imply dimension $A=n$. Thus by the induction hypothesis there exists $\bar{g} \in k\left[\bar{X}_{1}, \cdots, \bar{X}_{n-1}\right]$ such that

(a) $\bar{g}=\bar{X}_{n-1}-\bar{t}\left(\bar{X}_{1}, \cdots, \bar{X}_{n-2}\right)$,

(b) $(\bar{g}) \cap A=0$,

and in case $k$ is infinite, $\bar{t}$ can be taken to be linear. Let $g=X_{n-1}-$ $t\left(X_{1}, \cdots, X_{n-2}\right)$, then we have $(f, g) \cap A=P$. Now for each positive integer $\beta$ set $l_{\beta}=f-(g)^{\beta}$ (if $k$ is infinite set $l_{\lambda}=f-\lambda g$ for each $\lambda \in k$ ). Then $l_{i}$ has the form $X_{n}-d\left(X_{1}, \cdots, X_{n-1}\right)$ (in the infinite case $l_{\lambda}$ has the desired form). Now since $\left(l_{\beta}\right) \cap A \leqq P$ and each $l_{\beta}$ is prime we must have $\left(l_{\beta}\right) \cap A=P$ by the minimality of $P$. But if $P \neq 0$ let $a \neq 0 \in P$. Then $a$ has infinitely many prime divisors of form $l_{\beta}$, which is a contradiction. Thus $P=0$ and the lemma now follows easily.

Lemma C. If $k$ is a field and $X$ is an indeterminate over $k$ then if $A$ is any ring such that $k<A<k[X], A$ is an affine ring over $k$ whose integral closure is of the form $k[\theta]$.

Proof. It is known that any normal ring $A$ which is between $k$ and $k[X]$ is of the form $k[\theta][\mathbf{J}, \mathrm{p}$. 256]. Thus one can assume $X=\theta$. If $\theta \in k$ there is no problem. Let $f \in A-k$. Then $k[f]<A<k[\theta]$ since one could take $f=\theta^{n}+\alpha_{n-1} \theta^{n-1}+\cdots+\alpha_{1} \theta+\alpha_{0}, \theta$ is integral over $k[f]$. Thus $k[\theta]$ is a finite $k[f]$ module. Hence $A$ is also a finite $k[f]$ module which implies that $A$ is an affine ring over $k$.

THEOREM 1. Let $k$ be a field and $\left\{X_{\alpha}\right\}_{\alpha \in \Delta}$ a family of indeterminates over $k$. Let $A$ be a one-dimensional ring such that $k \leqq A \leqq k\left[\left\{X_{\alpha}\right\}_{\alpha \in \Delta}\right]$. Then $A$ is an affine ring over $k$. Moreover if $A$ is integrally closed then $A=k[\theta]$ for some $\theta$.

Proof. By Lemma $\mathrm{A}$ we reduce to the case of a finite number of indeterminates and by Lemma B we take this finite number to be one. The result then follows from Lemma $C$.

As a corollary to Lemmas A and B we have the following:

THEOREM 2. Let $k$ be a field, $\left\{X_{\alpha}\right\}_{\epsilon \Delta \alpha}$ a family of indeterminates over $k$ and $A$ an $n$ dimensional ring such that $k \leqq A \leqq k\left[\left\{X_{\alpha}\right\}_{\alpha \in \Delta}\right]$. Then the transcendence degree of $A$ over $k$ is exactly $n$. 
Proof. As in Lemma $\mathrm{B}$, dimension $A \leqq$ (transcendence degree of $A$ over $k$ ). But by Lemmas A and B (transcendence degree of $A$ over $k$ ) $\leqq$ dimension $A$.

THEOREM 3. Let $k$ be a field and $\left\{X_{\alpha}\right\}_{\alpha \in \Delta}$ a family of indeterminates over $k$. If $A$ is a ring which satisfies the d.c.c. on prime ideals and $k \leqq A \leqq$ $k\left[\left\{X_{\alpha}\right\}_{\alpha \in \Delta}\right]$ then there is an $a \in A$ such that $A[1 / a]$ is noetherian and the derived normal ring of $A[1 / a]$ is a finite $A[1 / a]$ module.

Proof. As in Theorem 1 we can assume $k \leqq A \leqq k\left[X_{1}, \cdots, X_{d}\right]$ where each $X_{i}$ is algebraic over $A$. Hence there is an $a \in A$ such that $k\left[1 / a, X_{1}, \cdots, X_{d}\right]$ is a finite $A[1 / a]$ module. Since $k\left[1 / a, X_{1}, \cdots, X_{d}\right]$ is noetherian, so is $A[1 / a]$ [E]. Since $k\left[1 / a, X_{1}, \cdots, X_{d}\right]$ is integrally closed we have the integral closure of $A[1 / a]$ is contained in $k\left[1 / a, X_{1}, \cdots, X_{d}\right]$. Hence it is a finite $A[1 / a]$ module.

REMARK. Let $k$ be a field and $X$ and $Y$ indeterminates over $k$. Let $A=k\left[\left\{X Y^{i}\right\}_{i=1}^{\infty}\right] \leqq k[X, Y]$. Then $A$ is not noetherian because the maximal ideal generated by $X Y, X Y^{2}, \cdots, X Y^{n}, \cdots$ is not finitely generated. In this example a suitable " $a$ " for Theorem 3 would be $X Y$.

Lemma D. Let $k\left(\theta_{1}, \cdots, \theta_{n}\right)$ be a finite extension of transcendence degree one over $k$. If $A$ is a domain such that $k \leqq A \leqq k\left(\theta_{1}, \cdots, \theta_{n}\right)$ then $A$ is a one-dimensional noetherian domain.

PROOF. This is an immediate corollary of the theorem of Krull-Akizuki [N, Theorem (33.2), p. 115].

THEOREM 4. Let $k$ be a field and $\left\{X_{\alpha}\right\}_{\alpha \in \Delta}$ a family of indeterminates over $k$. If $A$ is a two-dimensional Krull ring such that $k<A<k\left[\left\{X_{\alpha}\right\}_{\alpha \in \Delta}\right]$ then $A$ is noetherian.

Proof. As before we reduce to the case of two variables $X$ and $Y$ which are then algebraic over $A$. Choose $f$ and $g \in A$ such that $X$ and $Y$ are algebraic over $k[f, g]$. If necessary we can add a finite number of elements $\theta_{1}, \cdots, \theta_{k}$ such that $k\left[f, g, \theta_{1}, \cdots, \theta_{k}\right] \leqq A$, and $k\left[f, g, \theta_{1}, \cdots, \theta_{k}\right]$ has the same quotient field as $A$. But $A$ is a Krull overring of a twodimensional noetherian domain and hence $A$ is noetherian by a theorem of Heinzer $[\mathbf{H}]^{1}{ }^{1}$

${ }^{1}$ This theorem of Heinzer is extremely useful; our Theorem 4 is basically one of its simple applications. Professor Heinzer once observed to the author that there is a more elementary proof of this theorem than the one originally given in [H]. Indeed if one combines Theorem 3 of $[\mathbf{H}]$ with a result which now appears as an exercise in Kaplansky's Commutative rings [K, Exercise 8, p. 83] one obtains a relatively simple proof. Kaplansky sketches a proof of the exercise. In this exercise there is a minor misprint; the references to exercises in \$3-2 and §3-1 apparently should be to the corresponding exercises in $\S 2-2$ and $\S 2-1$. 
There is a much stronger version of Theorem 4 which uses a powerful theorem of Nagata. Since Theorem 4 can be done using considerably more elementary techniques, we have stated it separately.

THEOREM 5. Let $k$ be a field and $\left\{X_{\alpha}\right\}_{\alpha \in \Delta}$ a family of indeterminates over $k$. Suppose $A$ is a two-dimensional ring such that $k \subseteq A \subseteq k\left[\left\{X_{\alpha}\right\}_{\alpha \in \Delta}\right]$. Then $A$ is Krull if and only if $A$ is integrally closed and noetherian. Moreover $A$ is a normal affine ring over $k$ if and only if the residue field of every essential valuation of $A$ is transcendental over $k$.

Proof. The first equivalence is just Theorem 4. It is well known that an integrally closed noetherian domain is Krull [N, (33.4), p. 116]. Moreover if $A$ is a normal affine ring of dimension two over a field $k$, then the residue field of every essential valuation of $A$ is transcendental over $k$ [ZS, p. 92]. Thus we need only show that, with our hypothesis on the residue fields, $A$ is an affine ring. We proceed exactly as in Theorem 4, reducing two variables and finding a two-dimensional normal affine ring $B$ such that $B \subset A$ and $A$ and $B$ have the same quotient field. Now there is an $s \in B$ such that $k[x, y, 1 / s]$ is integral over $B[1 / s]$. Hence $A[1 / s]$ is integral over $B[1 / s]$ and since $B$ is normal, $A[1 / s]=B[1 / s]$. Consequently with possibly a finite number of exceptions, every essential valuation of $A$ is an essential valuation of $B$ (some of the essential valuations for $s$ as an element of $A$ may not be essential valuations of $B$ ). Let $V_{1}, \cdots, V_{n}$ denote this collection. It is a simple matter to choose a finite number of elements $c_{1}, \cdots, c_{t}$ in $A$ such that $B^{\prime}=B\left[c_{1}, \cdots, c_{t}\right]$ is a normal affine ring and has $V_{1}, \cdots, V_{n}$ as essential valuations. One chooses $c_{i} \in A$ such that the residue of $c_{i}$ is transcendental over $k$ in the residue field of $V_{i}$. Then each $V_{i}$ is centered on a minimal prime of $B^{\prime}$ and the $V_{i}$ are then essential valuations of $B^{\prime}$ (this is like the proof [ZS, Theorem 31, p. 89]). It then follows that $A$ is an 2 -transform of $B^{\prime}$, a normal affine ring of dimension two over $k$. Consequently $A$ is an affine ring $[\mathbf{N} ; 14 \mathrm{H}$, Theorem $4^{\prime}$, p. 53].

We close with an example showing that our Theorem 1 cannot be extended to rings of higher dimension.

EXAMPLE. Let $k[[t]]$ denote the formal power series in one variable over the field $k$ and let $x$ and $y$ be two elements of $k[[t]]$ which are algebraically independent over $k$ and have the same positive value. Let $V=k[[t]] \cap k(x, y)$ and $R=k[x, 1 / y] \cap V$. Then if $x=X$ and $1 / y=Y$ we have $k \subset k[X, X Y] \subset R \subset k[X, Y]$. The ring $R$ is Krull since it is the intersection of two Krull rings. But $V$ is an essential valuation of $R$ and the residue field of $V$ is $k$. Hence by Theorem $5, R$ is noetherian and $R$ is not an affine ring over $k$. 
ADDED IN PROOF. We are now aware of the fact that A. Zaks had already proven our Theorem 1 in his Dedekind subrings of $k\left[x_{1}, \cdots, x_{n}\right]$ are rings of polynomials, Israel J. Math. 9 (1971), 285-289.

\section{BIBLIOGRAPHY}

EZ. A. Evyatar and A. Zaks, Rings of polynomials, Proc. Amer. Math. Soc. 25 (1970), 559-562.

E. P. M. Eakin, Jr., The converse to a well known theorem on Noetherian rings, Math. Ann. 177 (1968), 278-282. MR 37 \#1360.

H. W. Heinzer, On Krull overrings of a Noetherian domain, Proc. Amer. Math. Soc. 22 (1969), 217-222. MR 40 \#7235.

J. N. Jacobson, Lectures in abstract algebra. Vol. 3: Theory of fields and Galois theory, Van Nostrand, Princeton, N.J., 1964. MR 30 \#3087.

K. I. Kaplansky, Commutative rings, Allyn and Bacon, Boston, Mass., 1970. MR 40 \#7234.

N. M. Nagata, Local rings, Interscience Tracts in Pure and Appl. Math., no. 13, Interscience, New York, 1962. MR 275790.

N; 14H. - Lectures on the fourteenth problem of Hilbert, Tata Institute of Fundamental Research Lectures on Math., no. 31, Tata Institute of Fundamental Research, Bombay, 1965. MR 35 \#6663.

ZS. O. Zariski and P. Samuel, Commutative algebra. Vol. II, University Series in Higher Math., Van Nostrand, Princeton, N.J., 1960. MR 22 \#11006.

Department of Mathematics, University of Kentucky, Lexington, Kentucky 40506 